Acta Crystallographica Section E

Structure Reports

Online

ISSN 1600-5368

\section{Francesco Punzo, ${ }^{\mathrm{a} *}$ ‡ David J.} Watkin, ${ }^{b}$ Sarah F. Jenkinson ${ }^{c}$ and George W. J. Fleet ${ }^{c}$

${ }^{\text {a}}$ Dipartimento di Scienze Chimiche, Facoltà di Farmacia, Università di Catania, Viale A. Doria 6, 95125, Catania, Italy, ${ }^{\mathbf{b}}$ Department of Chemical Crystallography, Chemical Research Laboratory, Mansfield Road, Oxford OX1 3TA, England, and ${ }^{\mathrm{c}}$ Department of Organic Chemistry, Chemical Research Laboratory, Mansfield Road, Oxford OX1 3TA, England

₹ Visiting Scientist at the Department of Chemical Crystallography, Chemical Research Laboratory, Mansfield Road, Oxford OX1 3TA, England.

Correspondence e-mail:

francesco.punzo@chemistry.oxford.ac.uk

\section{Key indicators}

Single-crystal X-ray study

$T=190 \mathrm{~K}$

Mean $\sigma(\mathrm{C}-\mathrm{C})=0.002 \AA$

$R$ factor $=0.031$

$w R$ factor $=0.078$

Data-to-parameter ratio $=12.0$

For details of how these key indicators were automatically derived from the article, see http://journals.iucr.org/e.

\title{
3,4-O-Isopropylidene-2-C-methyl-D-arabinono- 1,5-lactone
}

The title $\delta$-lactone, $\mathrm{C}_{9} \mathrm{H}_{14} \mathrm{O}_{5}$, formed in high diastereoselectivity by the Kiliani reaction of a protected 1-deoxyketose, adopts a boat conformation in which an $\mathrm{OH}$ group occupies a flagpole position.

\section{Comment}

Although sugars provide the largest group of readily available chiral building blocks and bioactive scaffolds (Lichtenthaler \& Peters, 2004), the potential of the Kiliani ascension of ketoses to provide readily available branched scaffolds has only just begun to be developed (Hotchkiss et al., 2004; Shallard-Brown et al., 2004 Cowley et al., 2004; van Ameijde et al., 2004). While the range of commercially available ketoses is restricted, 1-deoxyketoses may readily be generated by addition of organometallic reagents to sugar lactones. As an extension to the branching chemistry of ketoses, the protected 1-deoxyketose (1) was treated with sodium cyanide and gave a single diastereomeric product. The crystal structure reported in this paper firmly establishes that the lactone (2) was formed; none of the epimeric lactone (3) was isolated.

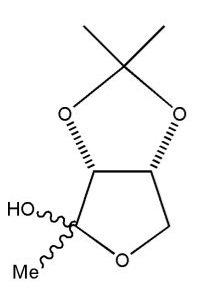

(1)

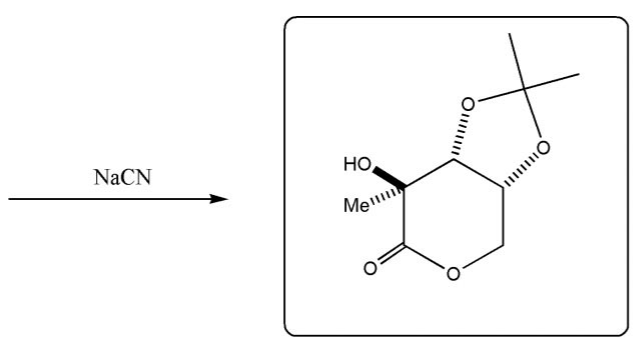

(2)
Received 23 November 2004 Accepted 9 December 2004 Online 18 December 2004<smiles>CC1(C)O[C@H]2COC(=O)[C@](C)(O)[C@H]2O1</smiles>

(3)
The $\delta$-lactone (2) (Fig. 1) adopts a boat conformation. While there are several example of fused 3,4-ketals of $\delta$-lactones that adopt boat conformations (Bruce et al., 1990; Bichard et al., 1991; Beacham et al., 1991), very few of them have a flagpole substituent (Wheatley et al., 1994); the hydroxy group at atom $\mathrm{C} 1$ is clearly in a very hindered position, being additionally attached to a tertiary $\mathrm{C}$ atom. Nonetheless, as usually expected for sugar derivatives, hydrogen bonding occurs between molecules (Fig. 2 and Table 2).

\section{Experimental}

The sugar was crystallized by dissolving it in diethyl ether, adding a few drops of cyclohexane and allowing the slow competitive evaporation of the two solvents until crystals formed. 


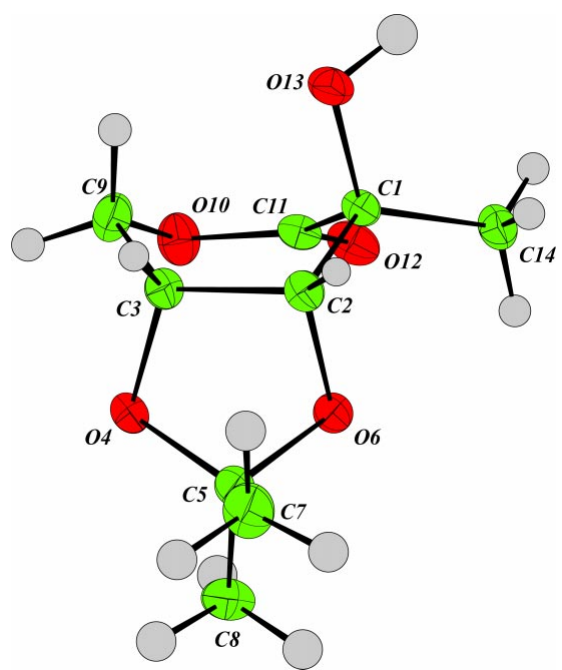

Figure 1

The molecular structure of (2), with displacement ellipsoids drawn at the $50 \%$ probability level. $\mathrm{H}$-atom radii are arbitrary.

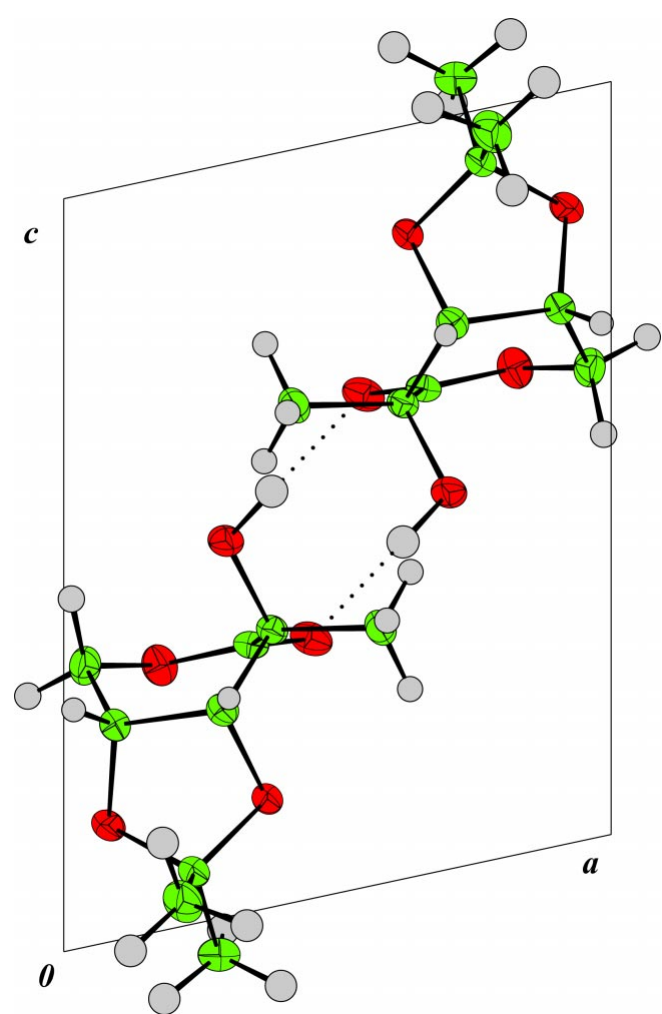

Figure 2

Partial packing diagram of (2), viewed down the $b$ axis. Hydrogen bonds are shown as dotted lines.

\section{Crystal data}

$\mathrm{C}_{9} \mathrm{H}_{14} \mathrm{O}_{5}$

$M_{r}=202.21$

Monoclinic, $P 2_{1}$

$a=7.7315(3) \AA$

$b=6.2859(3) \AA$

$c=10.4209(6) \AA$

$\beta=102.1024(17)^{\circ}$

$V=495.19(4) \AA^{3}$

$Z=2$
$D_{x}=1.356 \mathrm{Mg} \mathrm{m}^{-3}$

Mo $K \alpha$ radiation

Cell parameters from 1264 reflections

$\theta=5-30^{\circ}$

$T=190 \mathrm{~K}$

Needle, colourless

$0.90 \times 0.20 \times 0.20 \mathrm{~mm}$ $\mu=0.11 \mathrm{~mm}^{-1}$

\section{Data collection}

Nonius KappaCCD diffractometer

$\omega$ scans

2579 measured reflections

1527 independent reflections

1448 reflections with $I>2 \sigma(I)$

\section{Refinement}

Refinement on $F^{2}$

$R\left[F^{2}>2 \sigma\left(F^{2}\right)\right]=0.031$

$w R\left(F^{2}\right)=0.078$

$S=0.99$

1527 reflections

127 parameters

$\mathrm{H}$-atom parameters constrained

$$
\begin{aligned}
& R_{\text {int }}=0.018 \\
& \theta_{\max }=30.0^{\circ} \\
& h=-10 \rightarrow 10 \\
& k=-8 \rightarrow 8 \\
& l=-14 \rightarrow 14
\end{aligned}
$$

$w=1 /\left[\sigma^{2}\left(F^{2}\right)+0.04\right.$

$+0.06 P]$,

where $P=\left[\max \left(F_{\mathrm{o}}{ }^{2}, 0\right)+2 F_{\mathrm{c}}{ }^{2}\right] / 3$

$\Delta \rho_{\max }=0.24 \mathrm{e}^{-3}$

$\Delta \rho_{\min }=-0.16 \mathrm{e} \AA^{-3}$
$(\Delta / \sigma)_{\max }<0.001$

Table 1

Selected geometric parameters $\left(\AA{ }^{\circ}\right)$.

\begin{tabular}{llll}
\hline $\mathrm{C} 1-\mathrm{C} 2$ & $1.5284(18)$ & $\mathrm{O} 4-\mathrm{C} 5$ & $1.4312(16)$ \\
$\mathrm{C} 1-\mathrm{C} 11$ & $1.5318(19)$ & $\mathrm{C} 5-\mathrm{O} 6$ & $1.4314(16)$ \\
$\mathrm{C} 1-\mathrm{O} 13$ & $1.4334(15)$ & $\mathrm{C} 5-\mathrm{C} 7$ & $1.522(2)$ \\
$\mathrm{C} 1-\mathrm{C} 14$ & $1.5237(17)$ & $\mathrm{C} 5-\mathrm{C} 8$ & $1.508(2)$ \\
$\mathrm{C} 2-\mathrm{C} 3$ & $1.5468(18)$ & $\mathrm{C} 9-\mathrm{O} 10$ & $1.4581(18)$ \\
$\mathrm{C} 2-\mathrm{O} 6$ & $1.4261(16)$ & $\mathrm{O} 10-\mathrm{C} 11$ & $1.3367(16)$ \\
$\mathrm{C} 3-\mathrm{O} 4$ & $1.4268(16)$ & $\mathrm{C} 11-\mathrm{O} 12$ & $1.2124(17)$ \\
$\mathrm{C} 3-\mathrm{C} 9$ & $1.509(2)$ & & \\
$\mathrm{C} 2-\mathrm{C} 1-\mathrm{C} 11$ & $108.66(10)$ & $\mathrm{O} 4-\mathrm{C} 5-\mathrm{O} 6$ & $103.66(10)$ \\
$\mathrm{C} 2-\mathrm{C} 1-\mathrm{O} 13$ & $106.76(10)$ & $\mathrm{O} 4-\mathrm{C} 5-\mathrm{C} 7$ & $111.02(13)$ \\
$\mathrm{C} 11-\mathrm{C} 1-\mathrm{O} 13$ & $106.76(11)$ & $\mathrm{O} 6-\mathrm{C} 5-\mathrm{C} 7$ & $110.14(13)$ \\
$\mathrm{C} 2-\mathrm{C} 1-\mathrm{C} 14$ & $111.22(11)$ & $\mathrm{O} 4-\mathrm{C} 5-\mathrm{C} 8$ & $109.15(13)$ \\
$\mathrm{C} 11-\mathrm{C} 1-\mathrm{C} 14$ & $111.17(12)$ & $\mathrm{O} 6-\mathrm{C} 5-\mathrm{C} 8$ & $108.63(12)$ \\
$\mathrm{O} 13-\mathrm{C} 1-\mathrm{C} 14$ & $112.04(10)$ & $\mathrm{C} 7-\mathrm{C} 5-\mathrm{C} 8$ & $113.74(14)$ \\
$\mathrm{C} 1-\mathrm{C} 2-\mathrm{C} 3$ & $113.22(11)$ & $\mathrm{C} 5-\mathrm{O} 6-\mathrm{C} 2$ & $106.26(9)$ \\
$\mathrm{C} 1-\mathrm{C} 2-\mathrm{O} 6$ & $107.54(10)$ & $\mathrm{C} 3-\mathrm{C} 9-\mathrm{O} 10$ & $111.94(11)$ \\
$\mathrm{C} 3-\mathrm{C} 2-\mathrm{O} 6$ & $103.56(10)$ & $\mathrm{C} 9-\mathrm{O} 10-\mathrm{C} 11$ & $119.10(11)$ \\
$\mathrm{C} 2-\mathrm{C} 3-\mathrm{O} 4$ & $104.50(10)$ & $\mathrm{C} 1-\mathrm{C} 11-\mathrm{O} 10$ & $117.08(12)$ \\
$\mathrm{C} 2-\mathrm{C} 3-\mathrm{C} 9$ & $113.06(11)$ & $\mathrm{C} 1-\mathrm{C} 11-\mathrm{O} 12$ & $124.42(12)$ \\
$\mathrm{O} 4-\mathrm{C} 3-\mathrm{C} 9$ & $109.33(13)$ & $\mathrm{O} 10-\mathrm{C} 11-\mathrm{O} 12$ & $118.49(13)$ \\
$\mathrm{C} 3-\mathrm{O} 4-\mathrm{C} 5$ & $108.01(10)$ & &
\end{tabular}

Table 2

Hydrogen-bond geometry $\left(\AA,^{\circ}\right)$.

\begin{tabular}{lllll}
\hline$D-\mathrm{H} \cdots A$ & $D-\mathrm{H}$ & $\mathrm{H} \cdots A$ & $D \cdots A$ & $D-\mathrm{H} \cdots A$ \\
\hline $\mathrm{O} 13-\mathrm{H} 3 \cdots \mathrm{O} 12^{\mathrm{i}}$ & 0.94 & 1.87 & $2.8103(14)$ & 175
\end{tabular}

Symmetry code: (i) $-x+1, y+\frac{1}{2},-z+1$.

The multi-scan technique (Otwinowski \& Minor, 1997) was used to correct for changes in the illuminated volume of the long needle crystal. In the absence of significant anomalous scattering effects, Friedel pairs were merged. The absolute configuration was assigned from the known configuration of the starting material in the synthesis. The $\mathrm{H}$ atoms were all located in a difference map, but those attached to $\mathrm{C}$ atoms were repositioned geometrically. The $\mathrm{H}$ atoms were initially refined with soft restraints on the bond lengths and angles to regularize their geometry $(\mathrm{C}-\mathrm{H}=0.97-1.01 \AA$ and $\mathrm{O}-\mathrm{H}=0.94 \AA)$, after which they were refined as riding, with $U_{\text {iso }}(\mathrm{H})=1.2 U_{\text {eq }}(\mathrm{C})$ for those bonded to $\mathrm{C}$ atoms, and $U_{\text {iso }}(\mathrm{H})=0.05 \AA^{2}$ for the hydroxy group.

Data collection: COLLECT (Nonius, 1997); cell refinement: DENZO/SCALEPACK (Otwinowski \& Minor, 1997); data reduction: DENZO/SCALEPACK; program(s) used to solve structure: SIR92 (Altomare et al., 1994); program(s) used to refine structure: CRYSTALS (Betteridge et al., 2003); molecular graphics: 
CAMERON (Watkin et al., 1996); software used to prepare material for publication: CRYSTALS.

\section{References}

Ameijde, J. van, Cowley, A. R., Fleet, G. W. J., Nash, R. J., Simone, M. I. \& Soengas, R. (2004). Acta Cryst. E60, o2140-02141.

Altomare, A., Cascarano, G., Giacovazzo, G., Guagliardi, A., Burla, M. C., Polidori, G. \& Camalli, M. (1994). J. Appl. Cryst. 27, 435-435.

Beacham, A. R., Bruce, I., Choi, S., Doherty, O., Fairbanks, A. J., Fleet, G. W. J., Skead, B. M., Peach, J. M., Saunders, J. \& Watkin, D. J. (1991). Tetrahedron Asymmetry, 2, 883-900.

Betteridge, P. W., Carruthers, J. R., Cooper, R. I., Prout, K. \& Watkin, D. J. (2003). J. Appl. Cryst. 36, 1487.

Bichard, C. J. F., Fairbanks, A. J., Fleet, G. W. J., Ramsden, N. G., Vogt, K., Doherty, O., Pearce, L. \& Watkin, D. J. (1991). Tetrahedron Asymmetry, 2, 901-912.
Bruce, I., Fleet, G. W. J., Girdhar, A., Haraldsson, M., Peach, J. M. \& Watkin, D. J. (1990). Tetrahedron, 46, 19-32.

Cowley, A. R., Fleet, G. W. J., Simone, M. I. \& Soengas, R. (2004). Acta Cryst. E60, 02142-02143.

Hotchkiss, D., Soengas, R., Simone, M. I., van Ameijde, J., Hunter, S., Cowley, A. R. \& Fleet, G. W. J. (2004). Tetrahedron Lett. 45, 9461-9464.

Lichtenthaler, F. W. \& Peters, S. (2004). Compt. Rend. Chim. 7, 6590.

Nonius (1997). COLLECT. Nonius BV, Delft, The Netherlands.

Otwinowski, Z. \& Minor, W. (1997). Methods in Enzymology, Vol. 276, Macromolecular Crystallography, Part A, edited by C. W. Carter Jr \& R. M. Sweet, pp. 307-326. New York: Academic Press.

Shallard-Brown, H. A., Harding, C. C., Watkin, D. J., Soengas, R., Skytte, U. P. \& Fleet, G. W. J. (2004). Acta Cryst. E60, o2163-02164.

Watkin, D. J., Prout, C. K. \& Pearce, L. J. (1996). CAMERON. Chemical Crystallography Laboratory, Oxford, England.

Wheatley, J. R., Beacham, A. R., Lilley, P. D., Watkin, D. J. \& Fleet, G. W. J. (1994). Tetrahedron Asymmetry, 5, 2523-2534. 


\section{supporting information}

Acta Cryst. (2005). E61, o127-o129 [https://doi.org/10.1107/S1600536804032659]

\section{3,4-O-Isopropylidene-2-C-methyl-D-arabinono-1,5-lactone}

Francesco Punzo, David J. Watkin, Sarah F. Jenkinson and George W. J. Fleet

3,4-O-Isoprpylidene-D-arabinono-1,5-lactone

Crystal data

$\mathrm{C}_{9} \mathrm{H}_{14} \mathrm{O}_{5}$

$M_{r}=202.21$

Monoclinic, $P 2_{1}$

$a=7.7315$ (3) $\AA$

$b=6.2859(3) \AA$

$c=10.4209(6) \AA$

$\beta=102.1024(17)^{\circ}$

$V=495.19$ (4) $\AA^{3}$

$Z=2$

$F(000)=216$

$D_{\mathrm{x}}=1.356 \mathrm{Mg} \mathrm{m}^{-3}$

Mo $K \alpha$ radiation, $\lambda=0.71073 \AA$

Cell parameters from 1264 reflections

$\theta=5-30^{\circ}$

$\mu=0.11 \mathrm{~mm}^{-1}$

$T=190 \mathrm{~K}$

Needle, colourless

$0.90 \times 0.20 \times 0.20 \mathrm{~mm}$

Data collection

Nonius KappaCCD

1448 reflections with $I>2 \sigma(I)$

diffractometer

$R_{\text {int }}=0.018$

Graphite monochromator

$\omega$ scans

$\theta_{\text {max }}=30.0^{\circ}, \theta_{\text {min }}=5.3^{\circ}$

$h=-10 \rightarrow 10$

2579 measured reflections

$k=-8 \rightarrow 8$

1527 independent reflections

$l=-14 \rightarrow 14$

Refinement

Refinement on $F^{2}$

Least-squares matrix: full

$R\left[F^{2}>2 \sigma\left(F^{2}\right)\right]=0.031$

$w R\left(F^{2}\right)=0.078$

$S=0.99$

1527 reflections

127 parameters

1 restraint

Primary atom site location: structure-invariant direct methods

Hydrogen site location: inferred from neighbouring sites

$\mathrm{H}$-atom parameters constrained

$w=1 /\left[\sigma^{2}\left(F^{2}\right)+0.04+0.06 P\right]$,

where $P=\left(\max \left(F_{\mathrm{o}}^{2}, 0\right)+2 F_{\mathrm{c}}{ }^{2}\right) / 3$

$(\Delta / \sigma)_{\max }=0.000343$

$\Delta \rho_{\max }=0.24$ e $\AA^{-3}$

$\Delta \rho_{\min }=-0.16$ e $\AA^{-3}$

Fractional atomic coordinates and isotropic or equivalent isotropic displacement parameters $\left(\AA^{2}\right)$

\begin{tabular}{lllll}
\hline & $x$ & $y$ & $z$ & $U_{\text {iso }} * U_{\text {eq }}$ \\
\hline C1 & $0.38045(16)$ & $0.7432(2)$ & $0.63155(12)$ & 0.0190 \\
C2 & $0.28987(16)$ & $0.8696(2)$ & $0.72414(13)$ & 0.0206 \\
C3 & $0.09390(16)$ & $0.8070(2)$ & $0.71356(13)$ & 0.0221 \\
O4 & $0.08151(12)$ & $0.7559(2)$ & $0.84474(9)$ & 0.0292 \\
C5 & $0.23824(17)$ & $0.8319(3)$ & $0.93106(13)$ & 0.0254 \\
O6 & $0.37167(12)$ & $0.81163(18)$ & $0.85511(9)$ & 0.0231
\end{tabular}




$\begin{array}{lllll}\text { C7 } & 0.2183(2) & 1.0639(3) & 0.96718(18) & 0.0372 \\ \text { C8 } & 0.2838(2) & 0.6857(3) & 1.04806(15) & 0.0382 \\ \text { C9 } & 0.03922(18) & 0.6164(3) & 0.62648(15) & 0.0269 \\ \text { O10 } & 0.17525(13) & 0.45193(18) & 0.64662(11) & 0.0274 \\ \text { C11 } & 0.34223(18) & 0.5063(2) & 0.64601(13) & 0.0207 \\ \text { O12 } & 0.45221(14) & 0.36653(18) & 0.65495(10) & 0.0273 \\ \text { O13 } & 0.29608(12) & 0.80258(19) & 0.50056(9) & 0.0261 \\ \text { C14 } & 0.57875(16) & 0.7863(3) & 0.65965(13) & 0.0244 \\ \text { H21 } & 0.3019 & 1.0247 & 0.7105 & 0.0248^{*} \\ \text { H31 } & 0.0179 & 0.9270 & 0.6816 & 0.0280^{*} \\ \text { H71 } & 0.3345 & 1.1210 & 1.0175 & 0.0488^{*} \\ \text { H72 } & 0.1220 & 1.0707 & 1.0178 & 0.0488^{*} \\ \text { H73 } & 0.1804 & 1.1428 & 0.8838 & 0.0488^{*} \\ \text { H81 } & 0.3965 & 0.7308 & 1.1071 & 0.0479^{*} \\ \text { H82 } & 0.1833 & 0.7025 & 1.0914 & 0.0479^{*} \\ \text { H83 } & 0.2915 & 0.5387 & 1.0159 & 0.0479^{*} \\ \text { H91 } & -0.0661 & 0.5480 & 0.6502 & 0.0333^{*} \\ \text { H92 } & 0.0135 & 0.6646 & 0.5337 & 0.0333^{*} \\ \text { H141 } & 0.6340 & 0.7072 & 0.5946 & 0.0310^{*} \\ \text { H142 } & 0.5908 & 0.9419 & 0.6521 & 0.0310^{*} \\ \text { H143 } & 0.6324 & 0.7393 & 0.7493 & 0.0310^{*} \\ \text { H3 } & 0.3800 & 0.8152 & 0.4478 & 0.0500^{*}\end{array}$

Atomic displacement parameters $\left(\AA^{2}\right)$

\begin{tabular}{lllllll}
\hline & $U^{11}$ & $U^{22}$ & $U^{33}$ & $U^{12}$ & $U^{13}$ & $U^{23}$ \\
\hline C1 & $0.0200(5)$ & $0.0201(6)$ & $0.0178(6)$ & $0.0008(5)$ & $0.0061(4)$ & $0.0015(4)$ \\
C2 & $0.0211(5)$ & $0.0193(6)$ & $0.0222(6)$ & $0.0010(5)$ & $0.0062(4)$ & $0.0002(5)$ \\
C3 & $0.0193(5)$ & $0.0257(6)$ & $0.0218(6)$ & $0.0004(5)$ & $0.0057(4)$ & $-0.0008(5)$ \\
O4 & $0.0230(4)$ & $0.0451(7)$ & $0.0214(5)$ & $-0.0088(5)$ & $0.0086(4)$ & $-0.0016(5)$ \\
C5 & $0.0200(5)$ & $0.0368(9)$ & $0.0204(6)$ & $-0.0006(6)$ & $0.0068(4)$ & $-0.0051(6)$ \\
O6 & $0.0194(4)$ & $0.0302(5)$ & $0.0205(4)$ & $0.0011(4)$ & $0.0063(3)$ & $-0.0042(4)$ \\
C7 & $0.0321(7)$ & $0.0436(10)$ & $0.0381(9)$ & $0.0032(7)$ & $0.0127(7)$ & $-0.0163(8)$ \\
C8 & $0.0360(7)$ & $0.0563(11)$ & $0.0230(7)$ & $0.0015(8)$ & $0.0075(6)$ & $0.0031(7)$ \\
C9 & $0.0201(5)$ & $0.0299(7)$ & $0.0297(7)$ & $-0.0024(6)$ & $0.0029(5)$ & $-0.0053(6)$ \\
O10 & $0.0261(5)$ & $0.0216(5)$ & $0.0355(5)$ & $-0.0041(4)$ & $0.0089(4)$ & $-0.0027(4)$ \\
C11 & $0.0252(6)$ & $0.0219(6)$ & $0.0162(5)$ & $0.0010(5)$ & $0.0071(4)$ & $-0.0002(5)$ \\
O12 & $0.0356(5)$ & $0.0228(5)$ & $0.0265(5)$ & $0.0061(4)$ & $0.0134(4)$ & $0.0020(4)$ \\
O13 & $0.0256(4)$ & $0.0331(5)$ & $0.0205(4)$ & $0.0043(5)$ & $0.0069(3)$ & $0.0080(4)$ \\
C14 & $0.0204(5)$ & $0.0275(7)$ & $0.0269(6)$ & $0.0003(6)$ & $0.0084(5)$ & $0.0015(6)$ \\
& & & & & &
\end{tabular}

Geometric parameters $\left(\AA,{ }^{\circ}\right)$

\begin{tabular}{llll}
\hline $\mathrm{C} 1-\mathrm{C} 2$ & $1.5284(18)$ & $\mathrm{C} 7-\mathrm{H} 72$ & 0.999 \\
$\mathrm{C} 1-\mathrm{C} 11$ & $1.5318(19)$ & $\mathrm{C} 7-\mathrm{H} 73$ & 0.990 \\
$\mathrm{C} 1-\mathrm{O} 13$ & $1.4334(15)$ & $\mathrm{C} 8-\mathrm{H} 81$ & 0.997 \\
$\mathrm{C} 1-\mathrm{C} 14$ & $1.5237(17)$ & $\mathrm{C} 8-\mathrm{H} 82$ & 0.983 \\
$\mathrm{C} 2-\mathrm{C} 3$ & $1.5468(18)$ & $\mathrm{C} 8-\mathrm{H} 83$ & 0.989
\end{tabular}




\begin{tabular}{|c|c|c|c|}
\hline $\mathrm{C} 2-\mathrm{O} 6$ & $1.4261(16)$ & $\mathrm{C} 9-\mathrm{O} 10$ & $1.4581(18)$ \\
\hline $\mathrm{C} 2-\mathrm{H} 21$ & 0.992 & C9-H91 & 0.996 \\
\hline $\mathrm{C} 3-\mathrm{O} 4$ & $1.4268(16)$ & C9-H92 & 0.993 \\
\hline $\mathrm{C} 3-\mathrm{C} 9$ & $1.509(2)$ & $\mathrm{O} 10-\mathrm{C} 11$ & 1.3367 (16) \\
\hline C $3-\mathrm{H} 31$ & 0.971 & $\mathrm{C} 11-\mathrm{O} 12$ & $1.2124(17)$ \\
\hline $\mathrm{O} 4-\mathrm{C} 5$ & $1.4312(16)$ & $\mathrm{O} 13-\mathrm{H} 3$ & 0.938 \\
\hline $\mathrm{C} 5-\mathrm{O} 6$ & $1.4314(16)$ & C14-H141 & 1.006 \\
\hline $\mathrm{C} 5-\mathrm{C} 7$ & $1.522(2)$ & $\mathrm{C} 14-\mathrm{H} 142$ & 0.987 \\
\hline $\mathrm{C} 5-\mathrm{C} 8$ & $1.508(2)$ & $\mathrm{C} 14-\mathrm{H} 143$ & 0.985 \\
\hline $\mathrm{C} 7-\mathrm{H} 71$ & 1.007 & & \\
\hline $\mathrm{C} 2-\mathrm{C} 1-\mathrm{C} 11$ & $108.66(10)$ & $\mathrm{H} 71-\mathrm{C} 7-\mathrm{H} 72$ & 113.1 \\
\hline $\mathrm{C} 2-\mathrm{C} 1-\mathrm{O} 13$ & $106.76(10)$ & $\mathrm{C} 5-\mathrm{C} 7-\mathrm{H} 73$ & 106.8 \\
\hline $\mathrm{C} 11-\mathrm{C} 1-\mathrm{O} 13$ & $106.76(11)$ & $\mathrm{H} 71-\mathrm{C} 7-\mathrm{H} 73$ & 110.2 \\
\hline $\mathrm{C} 2-\mathrm{C} 1-\mathrm{C} 14$ & $111.22(11)$ & $\mathrm{H} 72-\mathrm{C} 7-\mathrm{H} 73$ & 109.0 \\
\hline $\mathrm{C} 11-\mathrm{C} 1-\mathrm{C} 14$ & $111.17(12)$ & $\mathrm{C} 5-\mathrm{C} 8-\mathrm{H} 81$ & 110.5 \\
\hline $\mathrm{O} 13-\mathrm{C} 1-\mathrm{C} 14$ & $112.04(10)$ & $\mathrm{C} 5-\mathrm{C} 8-\mathrm{H} 82$ & 103.5 \\
\hline $\mathrm{C} 1-\mathrm{C} 2-\mathrm{C} 3$ & $113.22(11)$ & $\mathrm{H} 81-\mathrm{C} 8-\mathrm{H} 82$ & 111.0 \\
\hline $\mathrm{C} 1-\mathrm{C} 2-\mathrm{O} 6$ & $107.54(10)$ & $\mathrm{C} 5-\mathrm{C} 8-\mathrm{H} 83$ & 108.4 \\
\hline $\mathrm{C} 3-\mathrm{C} 2-\mathrm{O} 6$ & $103.56(10)$ & $\mathrm{H} 81-\mathrm{C} 8-\mathrm{H} 83$ & 111.6 \\
\hline $\mathrm{C} 1-\mathrm{C} 2-\mathrm{H} 21$ & 110.6 & $\mathrm{H} 82-\mathrm{C} 8-\mathrm{H} 83$ & 111.5 \\
\hline $\mathrm{C} 3-\mathrm{C} 2-\mathrm{H} 21$ & 111.1 & $\mathrm{C} 3-\mathrm{C} 9-\mathrm{O} 10$ & 111.94 (11) \\
\hline $\mathrm{O} 6-\mathrm{C} 2-\mathrm{H} 21$ & 110.6 & $\mathrm{C} 3-\mathrm{C} 9-\mathrm{H} 91$ & 109.5 \\
\hline $\mathrm{C} 2-\mathrm{C} 3-\mathrm{O} 4$ & $104.50(10)$ & $\mathrm{O} 10-\mathrm{C} 9-\mathrm{H} 91$ & 105.1 \\
\hline $\mathrm{C} 2-\mathrm{C} 3-\mathrm{C} 9$ & $113.06(11)$ & C3-C9-H92 & 108.5 \\
\hline $\mathrm{O} 4-\mathrm{C} 3-\mathrm{C} 9$ & $109.33(13)$ & $\mathrm{O} 10-\mathrm{C} 9-\mathrm{H} 92$ & 110.3 \\
\hline $\mathrm{C} 2-\mathrm{C} 3-\mathrm{H} 31$ & 109.9 & H91-C9-H92 & 111.5 \\
\hline $\mathrm{O} 4-\mathrm{C} 3-\mathrm{H} 31$ & 110.2 & $\mathrm{C} 9-\mathrm{O} 10-\mathrm{C} 11$ & $119.10(11)$ \\
\hline $\mathrm{C} 9-\mathrm{C} 3-\mathrm{H} 31$ & 109.7 & $\mathrm{C} 1-\mathrm{C} 11-\mathrm{O} 10$ & $117.08(12)$ \\
\hline $\mathrm{C} 3-\mathrm{O} 4-\mathrm{C} 5$ & $108.01(10)$ & $\mathrm{C} 1-\mathrm{C} 11-\mathrm{O} 12$ & $124.42(12)$ \\
\hline $\mathrm{O} 4-\mathrm{C} 5-\mathrm{O} 6$ & $103.66(10)$ & $\mathrm{O} 10-\mathrm{C} 11-\mathrm{O} 12$ & 118.49 (13) \\
\hline $\mathrm{O} 4-\mathrm{C} 5-\mathrm{C} 7$ & $111.02(13)$ & $\mathrm{C} 1-\mathrm{O} 13-\mathrm{H} 3$ & 110.4 \\
\hline $\mathrm{O} 6-\mathrm{C} 5-\mathrm{C} 7$ & $110.14(13)$ & $\mathrm{C} 1-\mathrm{C} 14-\mathrm{H} 141$ & 109.6 \\
\hline $\mathrm{O} 4-\mathrm{C} 5-\mathrm{C} 8$ & $109.15(13)$ & $\mathrm{C} 1-\mathrm{C} 14-\mathrm{H} 142$ & 105.6 \\
\hline $\mathrm{O} 6-\mathrm{C} 5-\mathrm{C} 8$ & $108.63(12)$ & $\mathrm{H} 141-\mathrm{C} 14-\mathrm{H} 142$ & 112.1 \\
\hline $\mathrm{C} 7-\mathrm{C} 5-\mathrm{C} 8$ & $113.74(14)$ & $\mathrm{C} 1-\mathrm{C} 14-\mathrm{H} 143$ & 109.6 \\
\hline $\mathrm{C} 5-\mathrm{O} 6-\mathrm{C} 2$ & $106.26(9)$ & $\mathrm{H} 141-\mathrm{C} 14-\mathrm{H} 143$ & 109.7 \\
\hline $\mathrm{C} 5-\mathrm{C} 7-\mathrm{H} 71$ & 110.2 & $\mathrm{H} 142-\mathrm{C} 14-\mathrm{H} 143$ & 110.2 \\
\hline $\mathrm{C} 5-\mathrm{C} 7-\mathrm{H} 72$ & 107.3 & & \\
\hline
\end{tabular}

Hydrogen-bond geometry $\left(\AA,{ }^{\circ}\right)$

\begin{tabular}{lllll}
\hline$D-\mathrm{H} \cdots A$ & $D-\mathrm{H}$ & $\mathrm{H} \cdots A$ & $D \cdots A$ & $D-\mathrm{H}^{\cdots} A A$ \\
\hline $\mathrm{O} 13-\mathrm{H} 3 \cdots \mathrm{O} 12^{\mathrm{i}}$ & 0.94 & 1.87 & $2.8103(14)$ & 175 \\
\hline
\end{tabular}

Symmetry code: (i) $-x+1, y+1 / 2,-z+1$. 